\title{
New Periodic Solutions for the Singular Hamiltonian System
}

\author{
Yi Liao \\ School of Business Administration, Southwestern University of Finance and Economics, Chengdu 611130, China \\ Correspondence should be addressed to Yi Liao; yiliao@swufe.edu.cn
}

Received 30 May 2014; Accepted 20 October 2014; Published 9 November 2014

Academic Editor: Jianqing Chen

Copyright (C) 2014 Yi Liao. This is an open access article distributed under the Creative Commons Attribution License, which permits unrestricted use, distribution, and reproduction in any medium, provided the original work is properly cited.

By use of the Cerami-Palais-Smale condition, we generalize the classical Weierstrass minimizing theorem to the singular case by allowing functions which attain infinity at some values. As an application, we study certain singular second-order Hamiltonian systems with strong force potential at the origin and show the existence of new periodic solutions with fixed periods.

\section{Introduction}

We are mainly interested in the existence of periodic solutions $t \mapsto x(t) \in \Omega$, with a prescribed period, of the second-order differential equation

$$
\ddot{x}=-V^{\prime}(t, x) \text {, }
$$

with $\Omega=\mathbb{R}^{N}-\{0\}(N \in \mathbb{N}, N \geq 2)$ and $V \in C^{1}(\mathbb{R} \times \Omega, \mathbb{R})$ where $V^{\prime}(t, \cdot)$ denotes the gradient of the function $V(t, \cdot)$ defined on $\Omega$. The study of the periodic solutions of such equations has a substantial literature with the works [1-21] of particular importance for our purpose.

In the 1975 paper of Gordon [10], variational methods were used to study periodic solutions of planar 2-body type problems under assumptions which have come to be known as Gordon's Strong Force condition $\left(V_{1}\right)$ :

$\left(V_{1}\right)$ there exists a neighborhood $\mathcal{N}$ of 0 and a function $U \in$ $C^{1}(\Omega, \mathbb{R})$ such that

$$
\begin{aligned}
& \text { (i) } \lim _{x \rightarrow 0} U(x)=-\infty \text {; } \\
& \text { (ii) }-V(t, x) \geq\left|U^{\prime}(x)\right|^{2} \text { for every } x \in \mathcal{N}-\{0\} \text { and } \\
& t \in[0, T] \text {; }
\end{aligned}
$$$$
\text { (iii) } \lim _{x \rightarrow 0} V(t, x)=-\infty \text {. }
$$

For 2-body type problems in $R^{N}(N \geq 2)$, one can see the works of Ambrosetti-Coti Zelati, Bahri-Rabinowitz, Greco, and other mathematicians from [1-5, 18-21]. In this paper we wish to highlight two main results among them, that is, Theorems 1 and 2 . Firstly, we must specify three separate conditions $V(t, x)$ may satisfy about its behavior at infinity. Suppose that $V(t, x)$ is $T$-periodic in $t$; then

$\left(V_{2}\right) \lim _{|x| \rightarrow \infty} V(t, x)=0, \lim _{|x| \rightarrow \infty} V^{\prime}(t, x)=0$ (uniformly for $t$ ) and $V(t, x)<0$ for every $t \in[0, T]$, $x \in \Omega$;

$\left(V_{3}\right)$ there exist $c_{1}, M_{1}, R_{1}, v>0$ such that, for every $t \epsilon$ $[0, T]$ and $x \in \mathbb{R}^{N}$ with $|x| \geq R_{1}$,

(i) $\left|V^{\prime}(t, x)\right| \leq M_{1}$;

(ii) $V(t, x) \geq c_{1}|x|^{\nu}$;

$\left(V_{4}\right)$ there exist $c_{1}, R_{1}>0, \theta>1 / 2, v>1$ such that, for every $t \in[0, T],|x| \geq R_{1}$,

(i) $\theta V^{\prime}(t, x) x \leq V(t, x)$;

(ii) $V(t, x) \geq c_{1}|x|^{\nu}$.

Set $K=\left\{x \in \Omega \mid V^{\prime}(t, x)=0\right.$ for every $\left.t \in[0, T]\right\}$; there hold the following results.

Theorem 1 (Greco [11]). If $K=\emptyset$ and $\left(V_{1}\right)$ and one of $\left(V_{2}\right)-\left(V_{4}\right)$ hold, then in (1) there is at least one nonconstant $T$ periodic $C^{2}$ solution.

Theorem 2 (Bahri-Rabinowitz [3] and Greco [11]). Suppose $\partial V / \partial t \equiv 0$, so that $V(t, x) \equiv V(x)$, and the following condition holds:

$\left(V_{5}\right) K$ is compact (or empty).

If $\left(V_{1}\right)$ and one of $\left(V_{2}\right)-\left(V_{4}\right)$ hold, then in (1) there exist infinitely many nonconstant $T$-periodic $C^{2}$ solutions.

In this paper, we prove the following new theorem. 
Theorem 3. Suppose $V \in C^{1}(\mathbb{R} \times \Omega, \mathbb{R})$ satisfies condition $\left(V_{1}\right)$ and conditions

(C2) for the given $T>0, V(t+T, x)=V(t, x)$;

(C3) there exists $A>0$ such that, $\forall(t, x) \in \mathbb{R} \times \Omega$,

$$
3 V(t, x)-V^{\prime}(t, x) x \leq A
$$

(C4) $V(t, x) \rightarrow+\infty$ as $|x| \rightarrow+\infty$ uniformly for $0 \leq t \leq$ $T$.

Then the system (1) has a $T$-periodic solution.

Corollary 4. Suppose $\alpha \geq 2, \beta \geq 3, c_{1}>0, c_{2}>0, V \in$ $C^{1}(\Omega, \mathbb{R})$, and

$$
\begin{aligned}
& V(x)=-c_{1}|x|^{-\alpha}, \quad \text { for } 0<|x| \leq r_{1} \\
& V(x)=c_{2}|x|^{\beta}, \quad \text { for }|x| \geq r_{2}>r_{1}
\end{aligned}
$$

then, $\forall T>0$, the system (1) has a T-periodic solution. From the above example, we see that our potential does not satisfy any of conditions $\left(V_{2}\right),\left(V_{3}\right)$, and $\left(V_{4}\right)$.

\section{A Few Lemmas}

In order to prove Theorem 3, we will need to recall the following useful lemmas.

Lemma 5 (Sobolev-Rellich-Kondrachov [15]). It is well known that

$$
H^{1}=W^{1,2}\left(\frac{R}{T Z}, R^{N}\right) \subset C\left(\frac{R}{T Z}, R^{N}\right)
$$

and the imbedding is compact.

Lemma 6 (Eberlein-Shmulyan [15]). A Banach space $X$ is reflexive if and only if every bounded sequence in $X$ has a weakly convergent subsequence.

Lemma 7 (Ekeland [8]). Let $X$ be a Banach space, and suppose $\Phi$ defined on $X$ is Gateaux-differentiable, lower semicontinuous, and bounded from below. Then there is a sequence $\left\{x_{n}\right\}$ such that

$$
\Phi\left(x_{n}\right) \longrightarrow \inf \Phi, \quad\left(1+\left\|x_{n}\right\|\right)\left\|\Phi^{\prime}\left(x_{n}\right)\right\| \longrightarrow 0 .
$$

Definition 8 (see [8]). Let $X$ be a Banach space and $f \in$ $C^{1}(X, R)$. We say $f$ satisfies the $(P S)_{c}$ condition if whenever $\left\{x_{n}\right\} \subset X$ such that

$$
f\left(x_{n}\right) \longrightarrow c \text { with } f^{\prime}\left(x_{n}\right) \longrightarrow 0,
$$

then $\left\{x_{n}\right\}$ has a strongly convergent subsequence.

Interestingly, Cerami [22] considers a weaker compact condition on a Banach space than the classical $(P S)_{c}$ condition. Here we introduce a similar condition in an open subset of a Banach space.
Definition 9 (see [8]). Let $X$ be a Banach space; $\Lambda$ is an open subset; and suppose $\Phi$ defined on $\Lambda$ is Gateaux-differentiable. We say that $\phi$ satisfies the $(C P S)_{c}$ condition if whenever $\left\{x_{n}\right\}$ is a sequence such that

$$
\Phi\left(x_{n}\right) \longrightarrow c \text { with }\left(1+\left\|x_{n}\right\|\right)\left\|\Phi^{\prime}\left(x_{n}\right)\right\| \longrightarrow 0,
$$

then $\left\{x_{n}\right\}$ has a strongly convergent subsequence in $\Lambda$.

With this definition, we can deduce a minimizing result in an open subset of a Banach space, the proof of which is similar to the standard one.

Lemma 10 (see Mawhin-Willem [15]). Let $X$ be a Banach space, $\Lambda \subset X$ an open subset, and $f \in C^{1}(\Lambda, R)$. Assume $f$ has a lower bound on the closure $\bar{\Lambda}$ of $\Lambda$, and let $C=\inf _{x \in \bar{\Lambda}} f(x)$. If $f$ satisfies $(C P S)_{C}$ on $\Lambda \subset X$ and $f\left(x_{n}\right) \rightarrow+\infty$ as $x_{n} \rightarrow \partial \Lambda$, then $C$ is a critical value for $f$.

Lemma 11 (see [10]). Let $\Omega=\mathbb{R}^{N}-\{0\}(N \in \mathbb{N}, N \geq 2)$, and $V \in C^{1}(\mathbb{R} \times \Omega, \mathbb{R})$ satisfies the Gordon's Strong Force condition $\left(V_{1}\right)$; let

$$
\begin{gathered}
H^{1}=\left\{q: R \longrightarrow R^{N} \mid q \in L^{2}, \dot{q} \in L^{2}, q(t+T)=q(t)\right\}, \\
\Lambda=\left\{q \in H^{1}, q(t) \neq 0, \forall t\right\} .
\end{gathered}
$$

Define

$$
f(q)=\frac{1}{2} \int_{0}^{T}|\dot{q}|^{2} d t-\int_{0}^{T} V(t, q) d t, \quad q \in \Lambda .
$$

Then $f\left(x_{n}\right) \rightarrow-\infty$ as $x_{n} \rightarrow \partial \Lambda$.

\section{The Proof of Theorem 3}

Let $H^{1}=\left\{q: R \rightarrow R^{N} \mid q \in L^{2}, \dot{q} \in L^{2}, q(t+T)=q(t)\right\}$ and $\Lambda=\left\{q \in H^{1}, q(t) \neq 0, \forall t\right\}$.

Lemma 12 (see [2]). Suppose $V \in C^{1}(\mathbb{R} \times \Omega, \mathbb{R})$ satisfies condition (C2) and define

$$
f(q)=\frac{1}{2} \int_{0}^{T}|\dot{q}|^{2} d t-\int_{0}^{T} V(t, q) d t, \quad q \in \Lambda .
$$

Then the critical point of $f(q)$ is a T-periodic solution of (1).

Lemma 13. If $V$ satisfies $\left(V_{1}\right)$ and $(C 2)-(C 4)$ in Theorem 1 , then $f$ satisfies the Cerami-Palais-Smale condition for any $c \geq$ 0 ; that is, for any $\left\{x_{n}\right\} \subset \Lambda$, if

$$
f\left(x_{n}\right) \longrightarrow c, \quad\left(1+\left\|x_{n}\right\|\right) f^{\prime}\left(x_{n}\right) \longrightarrow 0,
$$

then $\left\{x_{n}\right\}$ has a strongly convergent subsequence and the limit is in $\Lambda$.

Proof. By condition $\left(V_{1}\right)$ and Lemma 11, we must have $f\left(x_{n}\right) \rightarrow+\infty$ as $x_{n} \rightarrow \partial \Lambda$. Since $f\left(x_{n}\right) \rightarrow c$, we know that, for any given $\epsilon>0$, there exists $N$ such that when $n>N$, there holds the inequality

$$
\frac{1}{2} \int_{0}^{T}\left|\dot{x}_{n}\right|^{2} d t-\int_{0}^{T} V\left(t, x_{n}\right) d t \leq c+\epsilon .
$$


The limit $\left(1+\left\|x_{n}\right\|\right) f^{\prime}\left(x_{n}\right) \rightarrow 0$ implies

$$
f^{\prime}\left(x_{n}\right) x_{n} \longrightarrow 0
$$

and so

$$
\begin{aligned}
& 2 f\left(x_{n}\right)+\int_{0}^{T}\left[2 V\left(t, x_{n}\right)-V^{\prime}\left(t, x_{n}\right) x_{n}\right] d t \\
& =f^{\prime}\left(x_{n}\right) x_{n} \longrightarrow 0 .
\end{aligned}
$$

Using condition (C3) together with the limits and inequalities (11), (12), and (14), we can choose $d>0$ such that when $n$ is large enough, there holds

$$
\int_{0}^{T}\left|\dot{x}_{n}\right|^{2} d t \leq d
$$

which implies $\int_{0}^{T}\left|\dot{x}_{n}\right|^{2} d t$ is bounded.

In the following we prove that $\left|x_{n}(0)\right|$ is bounded; otherwise, there is a subsequence, still denoted by $\left\{x_{n}(0)\right\}$, such that

$$
\left|x_{n}(0)\right| \longrightarrow+\infty \text {. }
$$

Then by Newton-Leibniz's formula, we have

$$
\min _{0 \leq t \leq 1}\left|x_{n}(t)\right| \geq\left|x_{n}(0)\right|-\left\|\dot{x}_{n}\right\|_{L^{2}} \longrightarrow+\infty .
$$

Now, by (C3) and (C4), we have

$$
2 V\left(t, x_{n}(t)\right)-V^{\prime}\left(t, x_{n}(t)\right) \cdot x_{n}(t) \longrightarrow-\infty,
$$

which contradicts the limit (14).

Hence, $\left\|x_{n}\right\|_{H^{1}}=\left\|\dot{x}_{n}\right\|_{L^{2}}+\left|x_{n}(0)\right|$ is bounded; $\left\{x_{n}\right\}$ has a weakly convergent subsequence; we still denote it by $x_{n}$, and let the limit be $x$. We can show in a standard fashion that this subsequence is strongly convergent in $H^{1}$. To complete the proof, we write it out.

Since the sequence $x_{n}$ is bounded in $H^{1}$, so, by Sobolev's embedding inequality, we know it is also bounded in maximum norm, and, by condition $\left(V_{1}\right)$ and Lemma 11, we know that when $n$ is large,

$$
\max _{0} \leq t \leq T\left|x_{n}(t)\right|>0 .
$$

By $V \in C^{1}(\mathbb{R} \times \Omega, \mathbb{R})$, when $n$ is large, $V^{\prime}\left(x_{n}(t)\right)$ is also uniformly bounded in maximum norm; we have

$$
f^{\prime}\left(x_{n}\right) y=\int_{0}^{T}\left[\dot{x}_{n} \dot{y}-V^{\prime}\left(t, x_{n}\right) y\right] d t
$$

Taking $y=x$ and $y=x_{n}$ in the above equation, we get

$$
\begin{aligned}
f^{\prime}\left(x_{n}\right) x & =\int_{0}^{T}\left[\dot{x}_{n} \dot{x}-V^{\prime}\left(t, x_{n}\right) x\right] d t, \\
f^{\prime}\left(x_{n}\right) x_{n} & =\int_{0}^{T}\left[\dot{x}_{n} \dot{x}_{n}-V^{\prime}\left(t, x_{n}\right) x_{n}\right] d t .
\end{aligned}
$$

Since $f^{\prime}\left(x_{n}\right) \rightarrow 0$, hence $f^{\prime}\left(x_{n}\right) x \rightarrow 0$; furthermore, since $x_{n}$ is bounded, so $f^{\prime}\left(x_{n}\right) x_{n} \rightarrow 0$. Hence, by (21) and the uniformly bounded property for $V^{\prime}\left(x_{n}(t)\right)$, we have

$$
\lim _{n \rightarrow \infty} \int_{0}^{T} \dot{x}_{n} \dot{x} d t=\lim _{n \rightarrow \infty} \int_{0}^{T}\left|\dot{x}_{n}\right|^{2} d t .
$$

By $x_{n} \rightarrow x$ weakly, we have

$$
\int_{0}^{1} \dot{x}_{n} \dot{x} d t+\left|x_{n}(0) \cdot x(0)\right|=\int_{0}^{1}|\dot{x}|^{2} d t+|x(0)|^{2} .
$$

By Sobolev Embedding Theorem, $\left\{x_{n}\right\}$ has a subsequence, still denoted by $\left\{x_{n}\right\}$ subject to $x_{n}(0) \rightarrow x(0)$.

We notice

$$
\begin{aligned}
\left\|x_{n}-x\right\| & \\
= & \left(\int_{0}^{1}\left|\dot{x}_{n}-\dot{x}\right|^{2} d t\right)^{1 / 2}-\left|x_{n}(0)-x(0)\right| \\
= & \left(\int_{0}^{1}\left|\dot{x}_{n}\right|^{2} d t-2 \int_{0}^{1} \dot{x}_{n} \dot{x} d t+\int_{0}^{1}|\dot{x}|^{2} d t\right)^{1 / 2} \\
& -\left|x_{n}(0)-x(0)\right| \\
& \longrightarrow\left(\|\dot{x}\|_{L^{2}}^{2}-2\|\dot{x}\|_{L^{2}}^{2}+\|\dot{x}\|_{L^{2}}^{2}\right)^{1 / 2}+0=0 .
\end{aligned}
$$

That is, $x_{n} \rightarrow x$ strongly in $\Lambda$. Then by Lemma 10 the proof of Theorem 3 is complete.

\section{Conflict of Interests}

The author declares that there is no conflict of interests regarding the publication of this paper.

\section{Acknowledgment}

The author sincerely thanks the referee for his/her valuable comments and suggestions.

\section{References}

[1] A. Ambrosetti and V. C. Zelati, "Closed orbits of fixed energy for singular Hamiltonian systems," Archive for Rational Mechanics and Analysis, vol. 112, no. 4, pp. 339-362, 1990.

[2] A. Ambrosetti and V. Coti Zelati, Periodic Solutions of Singular Lagrangian Systems, Springer, 1993.

[3] A. Bahri and P. H. Rabinowitz, "A minimax method for a class of Hamiltonian systems with singular potentials," Journal of Functional Analysis, vol. 82, no. 2, pp. 412-428, 1989.

[4] V. Benci and F. Giannoni, "Periodic solutions of prescribed energy for a class of Hamiltonian systems with singular potentials," Journal of Differential Equations, vol. 82, no. 1, pp. 60-70, 1989.

[5] C. Carminati, E. Séré, and K. Tanaka, "The fixed energy problem for a class of nonconvex singular Hamiltonian systems," Journal of Differential Equations, vol. 230, no. 1, pp. 362-377, 2006.

[6] K.-C. Chang, Infinite-Dimensional Morse Theory and Multiple Solution Problems, Progress in Nonlinear Differential Equations and their Applications, 6, Birkhäuser, Basel, Switzerland, 1993. 
[7] M. Degiovanni and F. Giannoni, "Dynamical systems with Newtonian type potentials," Annali della Scuola Normale Superiore di Pisa, vol. 15, no. 3, pp. 467-494, 1988.

[8] I. Ekeland, Convexity Methods in Hamiltonian Mechanics, Springer, New York, NY, USA, 1990.

[9] E. Fadell and S. Husseini, "A note on the category of the free loop space," Proceedings of the American Mathematical Society, vol. 107, no. 2, pp. 527-536, 1989.

[10] W. B. Gordon, "Conservative dynamical systems involving strong forces," Transactions of the American Mathematical Society, vol. 204, pp. 113-135, 1975.

[11] C. Greco, "Periodic solutions of a class of singular Hamiltonian systems," Nonlinear Analysis. Theory, Methods \& Applications, vol. 12, no. 3, pp. 259-269, 1988.

[12] E. W. van Groesen, "Analytical mini-max methods for Hamiltonian brake orbits of prescribed energy," Journal of Mathematical Analysis and Applications, vol. 132, no. 1, pp. 1-12, 1988.

[13] G. Hardy, J. E. Littlewood, and G. Pólya, Inequalities, Cambridge University Press, 2nd edition, 1952.

[14] P. Majer, "Ljusternik-Schnirelmann theory with local PalaisSmale conditions and singular dynamical systems," Annales de l'Institut Henri Poincaré: Analyse Non Linéaire, vol. 8, no. 5, pp. 459-476, 1991.

[15] J. Mawhin and M. Willem, Critical Point Theory and Applications, Springer, 1989.

[16] L. Pisani, "Periodic solutions with prescribed energy for singular conservative systems involving strong forces," Nonlinear Analysis: Theory, Methods \& Applications, vol. 21, no. 3, pp. 167179, 1993.

[17] P. H. Rabinowitz, "A note on periodic solutions of prescribed energy for singular Hamiltonian systems," Journal of Computational and Applied Mathematics, vol. 52, no. 1-3, pp. 147-154, 1994.

[18] K. Tanaka, "A prescribed energy problem for a singular Hamiltonian system with a weak force," Journal of Functional Analysis, vol. 113, no. 2, pp. 351-390, 1993.

[19] K. Tanaka, "A prescribed-energy problem for a conservative singular Hamiltonian system," Archive for Rational Mechanics and Analysis, vol. 128, no. 2, pp. 127-164, 1994.

[20] K. Tanaka, "Periodic solutions for singular Hamiltonian systems and closed geodesics on non-compact Riemannian manifolds," Annales de l'Institut Henri Poincaré, vol. 17, no. 1, pp. 1-33, 2000.

[21] S. Terracini, "Multiplicity of periodic solution with prescribed energy to singular dynamical systems," Annales de l'Institut Henri Poincaré. Analyse Non Linéaire, vol. 9, no. 6, pp. 597-641, 1992.

[22] G. Cerami, "Un criterio di esistenza per i punti critici so variete illimitate," Istituto Lombardo, Accademia di Scienze e Lettere, Rendiconti. Scienze Matematiche e Applicazioni A, vol. 112, pp. 332-336, 1978. 


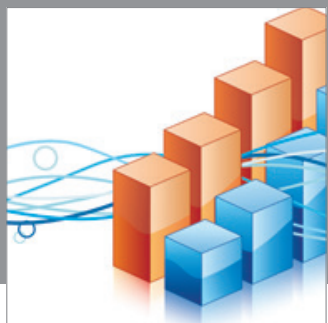

Advances in

Operations Research

mansans

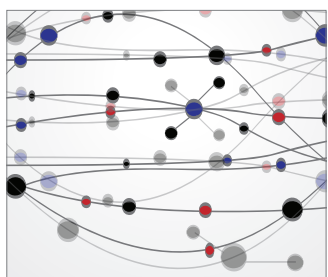

The Scientific World Journal
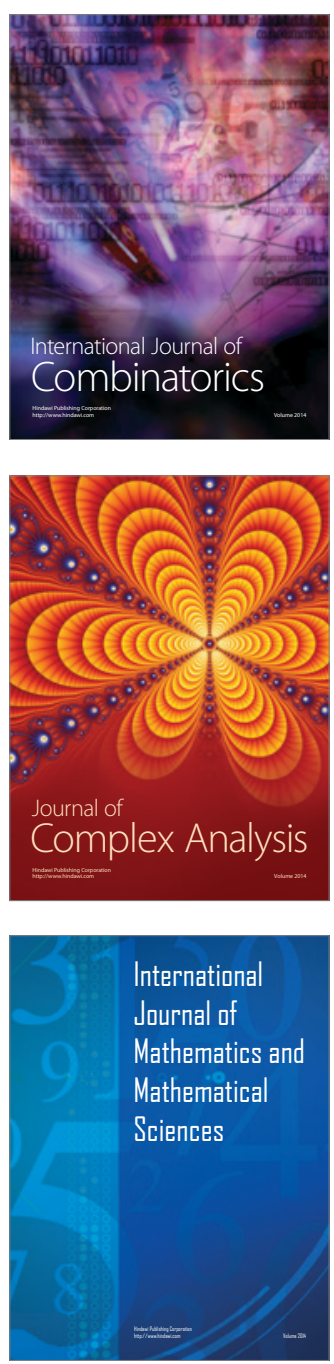
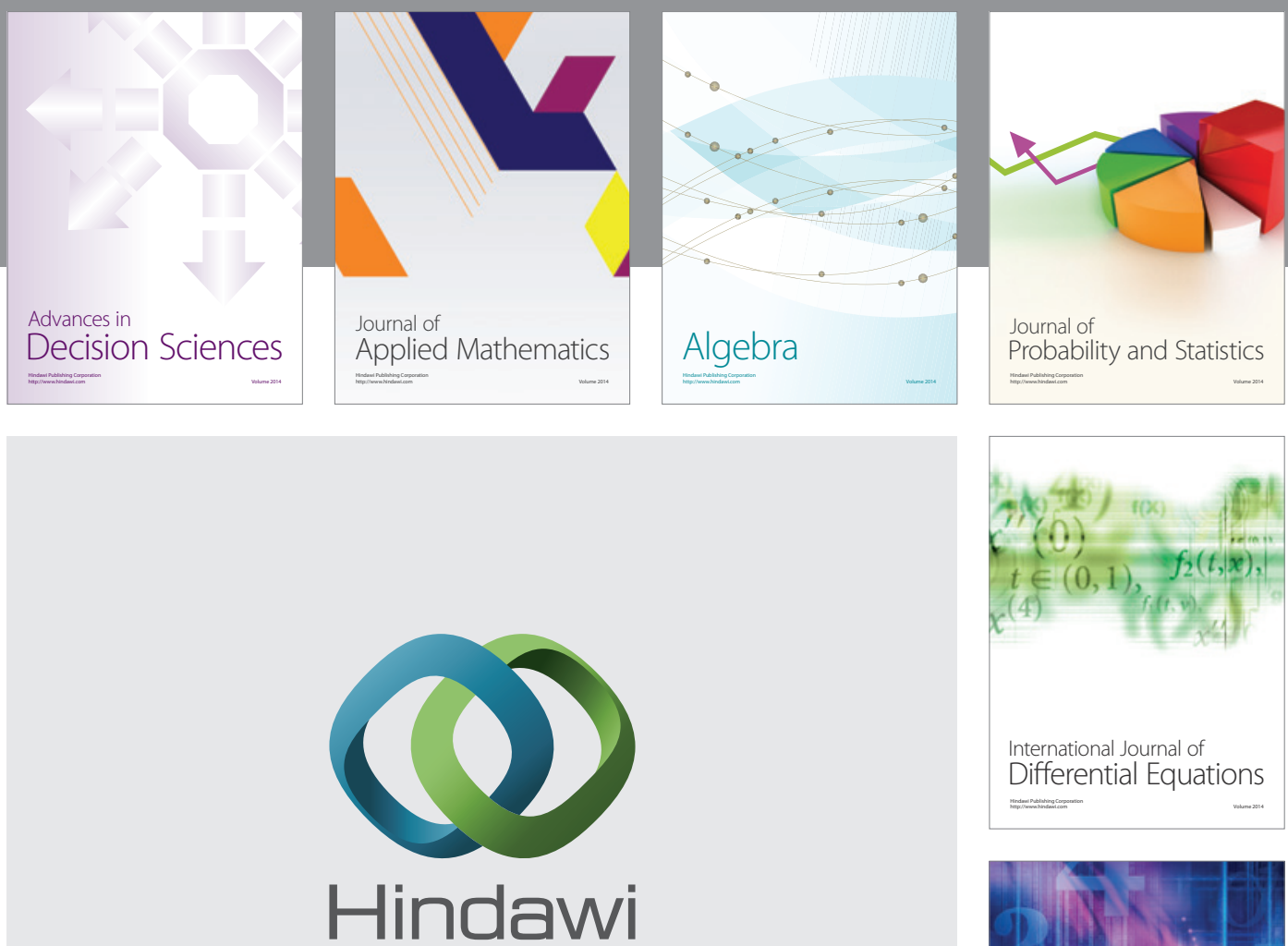

Submit your manuscripts at http://www.hindawi.com
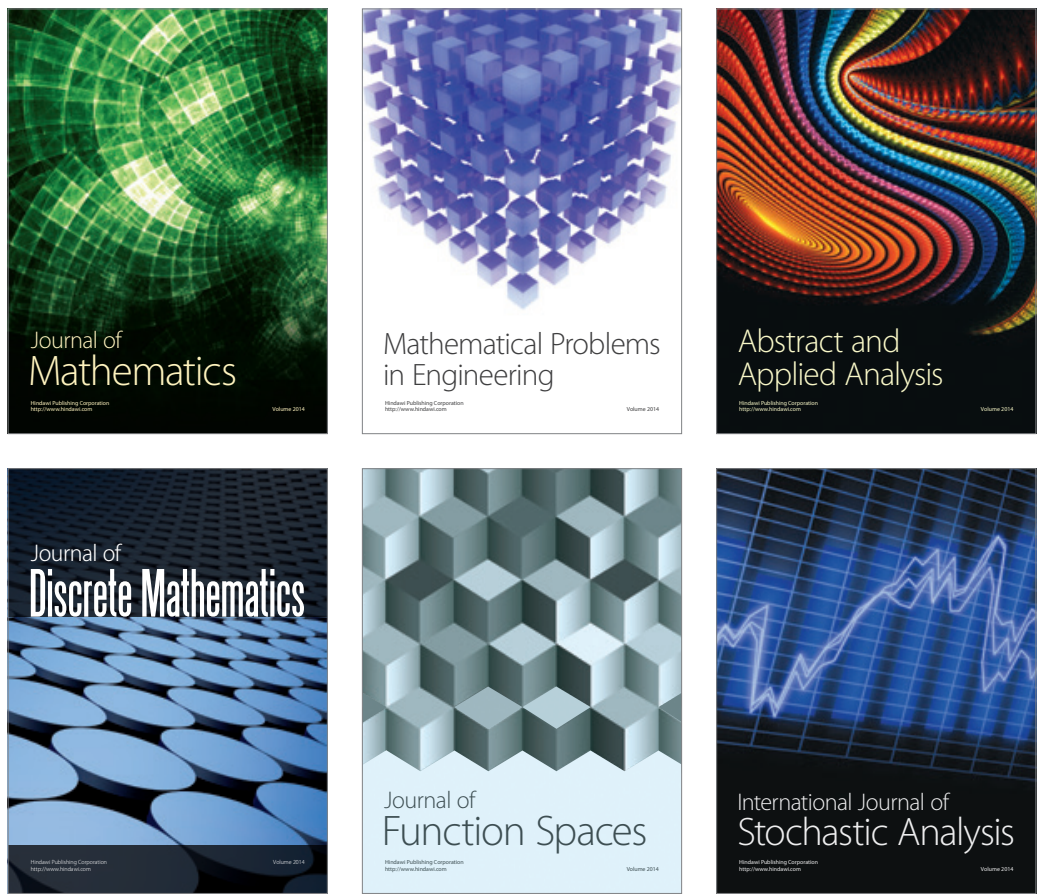

Journal of

Function Spaces

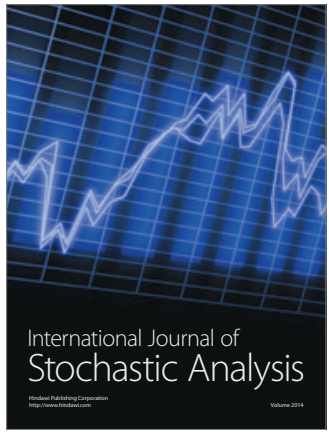

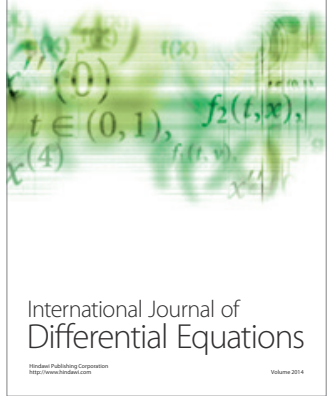
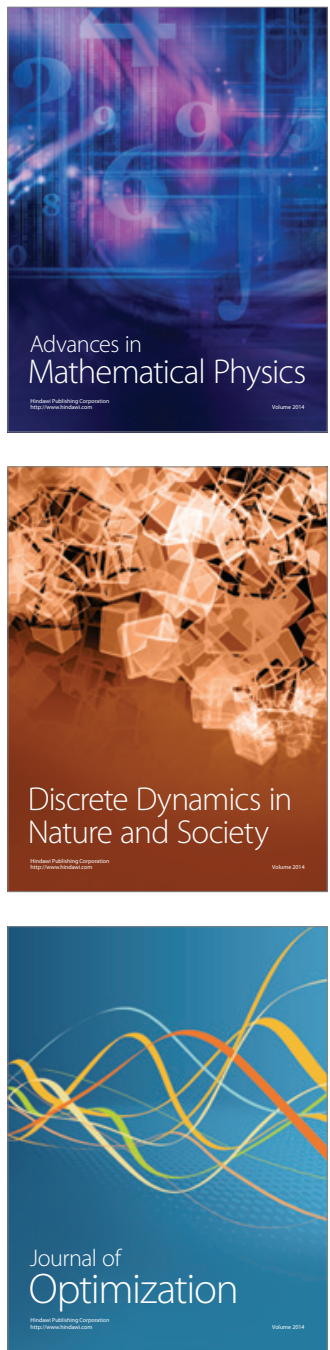